\title{
Psychoeducation an Effective Tool as Treatment Modality in Mental Health
}

\author{
Prashant Srivastava ${ }^{1 *}$, Rishi Panday ${ }^{2}$
}

\section{ABSTRACT}

Psychoeducation is an adjunctive approach which has the immense potentialities to reduce the relapse as well as rehospitalization rates and mental health costs in relapsing psychiatric disorders. Psychoeducation is understood as systematic, structured, didactic information on the illness and its treatment options and psychoeducation aims to enable patients as well as family members to cope with the illness. This paper shows that how psycho education is an effective tool as treatment modality in mental health. Psycho education has very important place in treatment and rehabilitation of patients with psychiatric disorders. Psychoeducation give directions to individuals with mental disorders and their family member about nature, course and prognosis about illness.

\section{Keywords: Psychoeducation, Effective Tool, Treatment and Mental Health}

Psychoeducation is an educative method based on clinical findings for providing information and training to families with psychiatrically ill persons to work together with mental health professionals as part of an overall clinical treatment plan for their ill family members. Psychoeducation has been shown to improve patient outcomes for persons with schizophrenia and other major mental illnesses and behavioral disorders. Imminent goals of psychoeducation are to prevent patients with severe mental illnesses from having frequent relapsing episodes of illness, ensuring medicine as well as treatment adherence, and to promote their re-entry into their home communities, with particular regard for their social and occupational functioning. To achieve these goals, psychoeducation programmes seek to provide families with the information they need about mental illness and the coping skills that will help them to deal with their loved one's psychiatric disorder. In a nutshell Psychoeducation's goal is to offer education and therapeutic strategies to improve the quality of life for the family while decreasing the possibility of relapse for the patient (Solomon, 1996). It also has been described as a "systematic didactic-

\footnotetext{
${ }^{1}$ Psychiatric Social Worker, CDC, Dept. of Pediatrics, MAMC and Ph.D Scholar, Dept. of Social Work, Jamia Millia Islamia, New Delhi, India

${ }^{2}$ Ph.D Scholar, Dept. of Social Work, Jamia Millia Islamia, New Delhi, India

*Responding Author

(C) 2016 Srivastava P, Panday R; licensee IJIP. This is an Open Access Research distributed under the terms of the Creative Commons Attribution License (http://creativecommons.org/licenses/by/2.0), which permits unrestricted use, distribution, and reproduction in any Medium, provided the original work is properly cited.
} 


\section{Psychoeducation an Effective Tool as Treatment Modality in Mental Health}

psychotherapeutic intervention, designed to inform patients and their relatives about the disorder and to promote coping" (Lincoln et al., 2007). By strengthening the coping skills, communication and problem solving abilities of the family, the well-being and adaptability of the individual and family members are expected to improve.

Definition of Psychoeducation: Psychoeducation can be defined as 'systematic, structured, didactic information on the illness and its treatment, and includes integrating emotional aspects in order to enable patients - as well as family members - to cope with the illness' (Bäuml \& Pitschel-Walz, 2008).

The term psychoeducation comprises systemic, didactic psychotherapeutic interventions, which are adequate for informing patients and their relatives about the illness and its treatment, facilitating both an understanding and personally responsible handling of the illness and supporting those afflicted in coping with the disorder” (Bäuml \& Pitschel-Walz, 2003).

Psychoeducation is the "process of teaching clients with mental illness and their family members about the nature of the illness, including its aetiology, progression, consequences, prognosis, treatment and alternatives” (Barker, 2003).

\section{Method of psychotherapeutic treatment:}

Educative method in health sector was used in as early as $18^{\text {th }}$ Century and early part of $19^{\text {th }}$ Century where philanthropists like Johann Heinrich Pestalozzi (1746-1827) and Dr. Samual Gridley Howe (1801-1876) used educative methods for providing therapeutic service and care to the physically and psychologically compromised people. But before the onset of "Mental Hygiene Movement" of early $20^{\text {th }}$ Century and "Deinstitutionalization Movement of 1950-60" there was no example of structured and organized psychoeducation. Psychoeducation came into the field of psychiatry strongly after the appearance of "Expressed Emotion" and "Family Burden Concept" in connection to severe and chronic psychiatric disorder like schizophrenia.

The known earliest evidence of the writings of psychoeducative intervention could be traced in the Journal of Abnormal Psychology: In that journal John E. Donley wrote about psychoeducation in an article namely "Psychotherapy and re-education", published in 1911. Subsequently Brian E. Tomlinson wrote a book entitled as: "The psychoeducational clinic" which was published by MacMillan Co in 1941. The popularization and development of the term psychoeducation into its current form can be attributed to the American researcher C.M. Anderson. She established this intervention as an adjunctive but effective treatment of schizophrenia in 1980. She made some commendable endeavours to scientifically establish psychoeducation as a medium of therapeutic intervention for the persons with chronic mental disorder like schizophrenia. She concentrated on educating relatives concerning the symptoms and the process of the schizophrenia. Also, her research focused on the stabilization of social authority and on the improvement in handling of the family members among themselves. Finally, 


\section{Psychoeducation an Effective Tool as Treatment Modality in Mental Health}

C.M. Anderson's research included more effective stress management techniques. Psychoeducation in behavior therapy has its origin in the patient's relearning of emotional and social skills. In the last few years increasingly systematic group programs have been developed, in order to make the knowledge more understandable to patients and their families (Hogarty et al., 1991; Baumlet al., 2006). In a single sentence psychoeducation could be explained as systematically used and structured forms of patient information, which are meant for informing the patient and/or the family members about the following things:

- Aetiology, factors (precipitating, predisposing and perpetuating) associated with the course and outcome of various mental disorders

- $\quad$ Signs and symptoms of mental disorders

- $\quad$ Explaining early signs of warning/triggering factors of relapses

- Guidance for the introspection and appropriate perception of typical symptoms of the problem

- How to act as a responsible person/how cope with the situation?

- When and whom to seek treatment?

- $\quad$ Dos and don'ts to family members while handling patient at home

- $\quad$ Dispelling off stigma, prejudices, misconception and negative attitudes to mental illness and patients

Therapeutic Factors of Psychoeducation: In psychoeducative intervention following therapeutic factors could be identified. Due to those factors psychoeducation becomes an effective tool to cater necessary information to patients and their key relatives as well as dispel negative attitudinal factors like expressed emotions and sense of burden (Baumlet al., 2006). Those are: Development of a good therapeutic relationship between patient and therapist, Unconditional appreciation towards patients, Empathic response to participants,_Respectful attention to subjectively deviant opinions, Need- and resource-orientated procedures, Stimulation of hope and reassurance to patients, Encouragement of personal exchange of experiences, Facilitation of "shared fate”' idea among the members (if group psychoeducation is initiated)

Models of psychoeducation: There are several different models of psychoeducation. These models include strategic elements while applying interventions like: development of single- and multiple-family groups; mixed groups that include family members and patients; groups of varying duration ranging from nine months to more than five years; and groups that focus on patients and families at different phases in the illness. The various psychoeducational models can be categorised into four approaches (Zipple \& Spanial, 1997). Most models used the component from more than one approach but usually they have specific focus.

- Information model: the emphasis of this model is to provide families the knowledge about psychiatric illness and its management. The aim of this approach is to improve the families' awareness about the illness and contribution to the management of the patient. 


\section{Psychoeducation an Effective Tool as Treatment Modality in Mental Health}

- The skill training model: this model is directed at systematically developing specific behaviours so that family members can enhance their capability to assist the ill relatives and manage the illness more effectively.

- The supportive model: it is an approach which generally utilizes support groups designed to engage the families of patient in sharing their feelings and experiences. Here the main goal is to enhance and improve the emotional capacities of the families to cope with the burden of caring for their ill relatives.

- Comprehensive model: it is also called combination approach because it consists of information, skill training and supportive model. In the initial phase of this approach members are given lectures about the illness. They are to take part in multi-family support group. In the final phase they have to participate particularly as a member of individual sessions with a mental health professional. (Left et al., 1992)

Family psychoeducation programs have been studied extensively and refined by a number of researchers, including Ian R.H. Falloon, Gerald Hogarty, William McFarlane, and Lisa Dixon. William McFarlane proposed a model of psychoeducation namely 'Multiple Family Group Therapy' (MFGT) with the aims of engaging families in the rehabilitation and after care programmes of severe psychiatric illness like schizophrenia. This model acknowledges the essentially chronic nature of this disease and seeks to engage families in the rehabilitation process by creating a long-term working partnership with them and providing them with the information needed to understand schizophrenia. This model seeks to assist the patient and family in accommodating the disease while developing social support systems for the reduction of confusing, anxiety, and exhaustion in the patient's family, while they learn adaptive strategies. William McFarlane delivered following assumptions of this model: Schizophrenia and schizoaffective disorders have strong biological correlates which point to the brain in the illness. This involvement can best be understood as a deficit in the attention-arousal mechanisms of the brain, yielding a condition whereby stimuli are idiosyncratically handled, Ordinary life events, and certainly extraordinary ones, can produce stress which overwhelms the patient's ability to respond in a directed and adaptive manner to a wide variety of stimuli, precipitating a psychotic episode, Schizophrenia is a chronic disease which is characterized by recurring episodes which each may last up to two years (including both positive and negative symptom phases), Families do not cause schizophrenia although they may inadvertently exacerbate the condition in their efforts to respond to it, The preferred response to the presence of schizophrenia is usually not deductible from "common sense" and may be in opposition to standard treatments for other mental disorders, Anti-psychotic and other psychotropic medications are generally of use in controlling symptoms although they are usually not sufficient in themselves to bring about complete rehabilitation, The use of street drugs or alcohol tends to exacerbate symptoms of schizophrenia, Clinicians who are warm, collegial, and non-blaming of families have the best chance of helping them learn new ways of responding to this illness, Clinicians need active support and supervision by more senior clinicians in their efforts to put this model into practice.

(c) The International Journal of Indian Psychology, ISSN 2348-5396 (e) | ISSN: 2349-3429 (p) | 126 


\section{Psychoeducation an Effective Tool as Treatment Modality in Mental Health}

\section{Peer-to-Peer Psychoeducation Approach:}

This approach was developed by Rummel et. al.(2005). According to them persons who underwent same kind of experience earlier can understand the problem of the people who have that problem now. Those people who had the problem earlier could empathize the problem of people who have recently develop that problem in much better manner than those people who never had that. In peer-to-peer psychoeducation programme mentally ill persons are given the access to mix with the people who had the same problem earlier but they recuperated from that problem. These people can motivate the patients up to considerable extent and provide them a new ray of hope. Rummel et. al. (2005) proposed a 5 step psychoeducation programme which can be delivered through peer educators who happen to the ex-patients. These ex-patients are called as 'peer moderators'. In step- 1 peer future peer-moderators are to take part in the meetings being conducted by the trained mental health professionals to know the subtle aspects of mental illness. Those peer-moderators have to provide education to the recently affected persons and their key caregivers. In step-2, 4 half day training workshops under the guidance of physicians and psychologists are conducted within a time span of 2 months. These future moderators at this stage gain the knowledge about conducting and managing group activities and also gained the thorough knowledge about psychiatric disorder (mainly schizophrenia as this method of psychoeducation is developed for schizophrenic patients). Training at this stage is done by video operated role playing and specific manuals which are specially developed for these peereducators or motivators. In step-3, 2 peer moderators are told to conduct group programmes for patients. A physician may be present at this stage but he has to take part in the group programme only when his intervention is required and he has to play a passive role at this stage. At step-4 peer moderators are to conduct psychoeducational group programmes independently under the passive supervision of mental health professionals. Group members, i.e., new patients are provided with information through workshops and group sessions. In step-5 recruitment of new peer moderators are done. At this step recovered patients who have the interest in providing proper information and knowledge about psychiatric disorder to recently ill people and their family members are included in this programme.

Short term goal directed psychoeducational programme is also developed in recent years. Researcher s had shown that this short term psychoeducational programme can be as beneficial as long term programmes. Bauml et al. (2006) showed that this brief, eight-session psychoeducation program may also have long-term effects like long term programme. They found statistically significant and clinically important differences between the persons who had received it and who hadn't. Their research was the part of the 7-year follow-up of the Psychosis Information Project Study (PIP-study). In this study separate groups for patients and their families were exmined. In Japan Yamaguchi et al (2006) postulated a short time goal directed psychoeducative intervention for the families with severely ill schizophrenia affected patients. Their therapy could be given in a period of 2 months and at the time of intervention the key relatives are to participate in three or four sessions of psychoeducation. Each session should last 


\section{Psychoeducation an Effective Tool as Treatment Modality in Mental Health}

for 2 hours and consist of two to six participants. The sessions are to be led by two or three doctors, a nurse and a social worker. These sessions of this approach should include interactive lectures on information about schizophrenia, rehabilitation, social support programs, and management of patient behaviors which should be followed by an intensive discussion, taking advantage of the small size of the groups. Authors claimed that after the intervention, both state and trait anxieties, subjective burden, sense of distress and depression of the relatives should come down at the significant level.

Pollio et al (2006) developed a format of composed but time limited single day psycho-education programme for relatives of patients with severe mental illnesses like schizophrenia, bipolar disorder, major depression, and other affective disorders. They had tested this approach of psychoeducation on few relatives of those patients. For that reason they organized a workshop for these people and saw the results. This 1-day psychoeducation workshop contains three lectures (descriptive and diagnostic information on schizophrenia and mood disorders, biological basis of mental illness, including neurochemistry and genetics, and medication and other treatment options). Informal discussion between the therapist and participants was organized during the lunch break of the work shop. After that two breakout sessions with a brief didactic presentation on following areas like 'area resources', 'success stories', 'ask the doc', 'religion', and 'legal rights' took place. They evaluated that short-term goals of the workshops were achieved satisfactorily. Expected positive goals of the workshop were 'control of daily life', 'effectiveness in crisis situation', 'knowledge on obtaining community resources', and 'knowledge about mental illness and treatment' and those things were significantly increased, whereas 'feelings of guilt' decreased. Authors opined that families with such patients would reap the fruits of this intervention in satisfactory manner.

\section{Passive \& Active Psychoeducation Models:}

Passive psychoeducational intervention is an educative as well as therapeutic intervention which provides information, education materials or feedback/advice. Examples of passive psychoeducation are programmes served to targeted individuals through means like leaflets, pamphlets, brochures, posters, audio-visual aids, lectures, internet material or software. This approach aims to educate the recipient about the nature and treatment of psychological distress. The intervention can be delivered in primary or secondary care settings, or within universities, community centres or other public venues. Passive psychoeducation programme does not require the recipient to undertake explicit homework or relaxation exercises and it does not deliver active treatment. Psychoeducation is often tagged with other active psychotherapies like CBT or IPT as an important part. This is not done with passive psychoeducative programme. Active psychoeducational intervention is the specific type of psychoeducation which is deemed as an important part of other psychotherapies like CBT, IPT, Family Therapy, Group Therapy or any other kinds of individual or group psychotherapies (Donker et al., 2009). 


\section{CONCLUSION}

The psychoeducation has been appeared as a major therapeutic approach to enable patients and their caregivers more skilful in dealing with the manifold stress being caused by psychiatric disorders. Psycho education has very important place in treatment and rehabilitation of patients with psychiatric disorders Over time, when individuals with serious mental illness and their families learn more about the illness and ways to reduce its effects, there can be many positive changes, such as: Fewer relapses, Less time spending in the psychiatric hospital, Decreased sense of stigma, Feeling of better control over life, Better medication adherence, Having better social living and problem solving skills among patients, Better global family functioning, Less occurrences of depression and anxiety among caregivers.

\section{Acknowledgments}

The author appreciates all those who participated in the study and helped to facilitate the research process.

\section{Conflict of Interests}

The author declared no conflict of interests.

\section{REFERENCES}

Bauml J, Pitschel-Walz G. Psychoedukation bei schizophrenen Erkrankungen. Stuttgart, Germany: Schattauer; 2003. Article in German. As cited in: J Bäuml, T Frobo“se, S Kraemer, M Rentrop, and G Pitschel-Walz (2006). Psychoeducation: A basic psychotherapeutic intervention for patients with schizophrenia and their families. Schizophrenia Bulletin, 32(1), S1-S9.

Bauml, J., Frobose, T., Kraemer, S., Rentrop, M. and Pitschel-Walz, G. (2006). Psychoeducation: A basic psychotherapeutic intervention for patients with schizophrenia and their families. Schizophrenia Bulletin, 32(1), S1-S9.

Bäumlet, Josef, et al. Psychoeducation: A Basic Psychotherapeutic Intervention for Patients with Schizophrenia and Their Families. Schizophrenia Bulletin. 200632 (Supplement 1): S1-S9.

Donker, T., Griffits, K.M., Cuijpers, P., Christensen, H. (2009). Psychoeducation for depression, anxiety and psychological distress: A meta-analysis. Child and Adolescent Psychiatry and Mental Health, 7:79.

Hogarty, G.E.; Anderson, CM.; Reiss, D.J.; Kornblith, S.J.; Greenwald, D.P.; Ulrich, R.F.; and Carter, M. (1991). Family psychoeducation, social skills training, and maintenance chemotherapy in the aftercare treatment of schizophrenia: II. Two-year effects of a controlled study on relapse and adjustment. Archives of General Psychiatry, 48, 340-347.

Leff, J.; Kuipers, L.; Berkowitz, R.; Eberlein-Vries, R.; and Sturgeon, D. (1992). A controlled trial of social intervention in the families of schizophrenic patients. British Journal of Psychiatry, 141, 121-134. 


\section{Psychoeducation an Effective Tool as Treatment Modality in Mental Health}

Lincoln, T.M., Wilhelm, K., Nestoriuc, Y. (2007). Effectiveness of psychoeducation for relapse, symptoms, knowledge, adherence and functioning in psychotic disorders: A metaanalysis. Schizophrenia Research, 96 (1-3), 232-245.

Pollio, D.E., North, C.S., Reid, D.L/, et al. (2006). Living with severe mental illness: What families and friends must know: Evaluation of a one-day psychoeducation workshop. Social Work, 51, 31-38. As cited in: C Rummel-Kluge \& W Kissling (2008). Psychoeducation in schizophrenia: New developments and approaches in the field. Current Opinion in Psychiatry, 21, 168-172.

Rummel, C.B., Hansen, W., Helbig, A., Pitschel-Walz, G., Kissling, W. (2005). Peer-to-peer psychoeducation in schizophrenia: a new approach. Journal of Clinical Psychiatry, 66, 1580-1585.

Solomon, P. (1996). Moving from psychoeducation for families of adults with serious mental illness. Psychiatric Services, 47 (12), 1364-1370.

Yamaguchi, H., Takahashi, A., Takano, A., Kojima, T. (2006). Direct effects of short-term psychoeducational intervention for relatives of patients with schizophrenia in Japan. Psychiatry and Clinical Neuroscience, 60, 590-597. As cited in: C Rummel-Kluge \& W Kissling (2008). Psychoeducation in schizophrenia: New developments and approaches in the field. Current Opinion in Psychiatry, 21, 168-172.

How to cite this article: Srivastava P, Panday R (2016), Psychoeducation an Effective Tool as Treatment Modality in Mental Health, International Journal of Indian Psychology, Volume 4, Issue 1, No. 82, ISSN:2348-5396 (e), ISSN:2349-3429 (p), DIP:18.01.153/20160401, ISBN:9781-365-59365-9 